\title{
A Cross Sectional Clinico Pathological Study of Diabetic Foot
}

\author{
Dr. Sapna $\mathrm{T}^{1^{*}}$
}

${ }^{1}$ MBBS, MS, Associate Professor, Department of General Surgery, Sapthagiri Institute of Medical Sciences \& Research Center, 15, Hesarghatta Main Rd, Navy Layout, Chikkasandra, Chikkabanavara, Bengaluru, Karnataka 560090, India

*Corresponding author: Dr. Sapna T

Abstract

A diabetic foot is any pathology that results directly from peripheral arterial disease (PAD) and/or sensory neuropathy that may affect the feet in diabetes mellitus; It presents as a long standing or rather chronic complication of diabetes mellitus. Diabetic foot syndrome encompasses a whole variety of diabetic foot pathological manifestations such as infection, Diabetic foot ulcer and neuropathic osteoartropathy [1, 2]. This study was conducted comprising of 25 patients of Diabetic foot in the Department of Surgery at tertiary care centre. Management of diabetic ulcers frequently involves the use of an empiric antibiotic. The wound severity and local antimicrobial susceptibility pattern often determine the selection of empiric treatment.

Keywords: Diabetic Foot, Ulcer, Staphylococcus Aureus.

Copyright $(\mathcal{O} 2021$ The Author(s): This is an open-access article distributed under the terms of the Creative Commons Attribution 4.0 International License (CC BY-NC 4.0) which permits unrestricted use, distribution, and reproduction in any medium for non-commercial use provided the original author and source are credited.

\section{INTRODUCTION}

Diabetic foot is any pathology that results directly from peripheral arterial disease (PAD) and/or sensory neuropathy that may affect the feet in diabetes mellitus; It presents as a long standing or rather chronic complication of diabetes mellitus. Diabetic foot syndrome encompasses a whole variety of diabetic foot pathological manifestations such as infection, Diabetic foot ulcer and neuropathic osteoarthropathy [1].

Diabetic neuropathy associated with diabetes may lead to insensate foot due to increasing peripheral nerve dysfunction; consequently patients' feet may have a reduced ability to feel pain. As a result of compromised foot sensation, the minor wounds may remain undetected for longer periods of time that may eventually progress to full-thickness diabetic ulcer. The diabetic foot with pain insensitivity can be identified or detected by $512 \mathrm{~mm}$ quantitative pinprick stimulation. The ongoing Research predictions for the lifetime incidence of foot ulcers within the diabetic population is around $15 \%$ and may become as high as $25 \%$ [3, 4].

People with Diabetes may have a combination of peripheral neuropathy and peripheral arterial disease leading to poor blood perfusion to the extremities commonly known as diabetic angiopathy. It has been found that more than $50 \%$ of diabetic patients with foot ulcer have a concurrent peripheral Arterial disease. Another interesting observation made is that deficiency of Vitamin D IS ALSO recently been found to be a contributing factor for diabetic foot infections with increased risk of amputations and mortalities [5].

In diabetic foot as a result of delayed wound healing pattern, there is always a danger of new infections setting in and gradually involving the bones plus joints necessitating a lower limb amputation. Un resolving Foot infections or progressive diabetic foot ulcer is the leading cause of non-traumatic amputation in Diabetic community.

Diabetic foot PREVENTION is a multipronged approach that should have optimised glycemic control along with inclusion of adequate screening of people with diabetes having increased risk for foot ulcerations particularly those patients having severe peripheral neuropathy; Patient sensitisation and education aiming for self foot examination with adequate knowledge of recognising foot complications and foot care go a long way in reducing the incidences of foot ulcer. Patients are educated about foot inspection methods and to look for hyperkeratosis, fungal infection, skin lesions and foot deformities. Prescribing a adequate size footwear to minimize repeat injury from ill-fitting shoe ware in Diabetic patients with peripheral neuropathy also goes a long way in prevention. However there is limited evidence to show that patient education of minimal or lesser quality may have a preventive effect on the longer run. 
A meta-analysis revealed that foot temperature-guided avoidance therapy among all the preventive therapies was the most beneficial in RCTs.

OBJECTIVE: A Cross sectional ClinicoPathological study of Diabetic Foot.

\section{METHODOLOGY}

This study was conducted comprising of 25 patients of Diabetic foot in the Department of Surgery at tertiary care centre during July to Dec 2017.

\section{Inclusion Criteria}

- All patients in the age group of 30-80years

- Patients having known diabetic history and diagnosed diabetic on admission with a diabetic foot.

\section{Exclusion Criteria}

- Patients with systemic disease like IHD are not included in these study.

- Patients with venous ulcers are not included in the study

- Patients with chronic foot and leg ulcers due to causes other than diabetes, cellulitis, blisters, osteomyelitis, gangrene of lower limb due to causes other than diabetes.

\section{RESULTS}

Table-1: Age Wise Distribution of Cases

\begin{tabular}{|l|l|l|}
\hline Age Group & No. of Patients & Percentage (\%) \\
\hline $31-40$ & 00 & 00 \\
\hline $41-50$ & 11 & 44 \\
\hline $51-60$ & 09 & 36 \\
\hline $61-70$ & 03 & 12 \\
\hline $71-80$ & 02 & 08 \\
\hline
\end{tabular}

Most of the diabetic patients were in the age group of 41-50 years followed by 51-60 year age group.

Table-2: Sex Wise Distribution of Cases

\begin{tabular}{|l|l|l|}
\hline Sex & No of patients & Percentage (\%) \\
\hline Male & 16 & 64 \\
\hline Female & 09 & 36 \\
\hline
\end{tabular}

Out of 25 cases studied, there was a male predominance in occurrence of diabetic lesions 64\% were male patients and $36 \%$ were female patients.

Table-3: Distribution of Cases Based on Clinical Presentation

\begin{tabular}{|l|l|l|}
\hline Clinical presentation & No of Patients & Percentage (\%) \\
\hline Abscess & 2 & 08 \\
\hline Ulcer & 11 & 44 \\
\hline Cellulitis & 08 & 36 \\
\hline Gangrene & 04 & 14 \\
\hline
\end{tabular}

Most common presentation was ulcer $44 \%$ of cases followed by cellulitis in $36 \%$ of patients.
Gangrene was seen in $14 \%$ of patients and abscess was seen in $8 \%$ of patients.

Table-4: Distribution of Cases Based On Duration of Diabetes Mellitus

\begin{tabular}{|l|l|l|}
\hline Duration of DM & No of Patients & Percentage (\%) \\
\hline 1-5 years & 01 & 04 \\
\hline 6-10 years & 12 & 48 \\
\hline 11-15 years & 08 & 36 \\
\hline $16-20$ years & 04 & 16 \\
\hline
\end{tabular}

Most of the patient, 12 cases had diabetes duration for about 6-10 years (48\%), followed by 8 cases had 11-15 $(36 \%)$ years of diabetes duration.

Table-5: Distribution of Cases Based On Complications

\begin{tabular}{|l|l|l|}
\hline Complications & No of patients & Percentage (\%) \\
\hline Neuropathy & 12 & 48 \\
\hline Vasculopathy & 08 & 32 \\
\hline Both & 03 & 12 \\
\hline No Neuropathy/Vasculopathy & 02 & 8 \\
\hline
\end{tabular}

Neuropathy was the commonest present in patients (48\%), followed by vasculopathy (32\%) and $12 \%$ of cases presented with both complications. 
Table-6: Distribution of Cases Based On Causative Organisms

\begin{tabular}{|l|l|l|}
\hline Causative organism & No of Patients & Percentage (\%) \\
\hline Staph aureus & 11 & 44 \\
\hline Streptococcus & 08 & 36 \\
\hline Pseudomonas aerginosa & 03 & 12 \\
\hline E coli & 01 & 4 \\
\hline Klebsilla & 01 & 4 \\
\hline Proteus & 01 & 4 \\
\hline
\end{tabular}

The most common microorganism grown on culture of pus was staphylococcus aureus in 11 patients followed by Streptococcus 8, pseudomonas 3, E coli 1, Proteus1.

\section{DISCUSSION}

In the present study, the vast majority of diabetic patients were in the age category of 41-50 years followed by 51-60 year age category. Out of 25 cases studied, there was a male predominance in occurrence of diabetic lesions $64 \%$ were male patients and 36\%were female patients. Most common presentation was ulcer $44 \%$ of cases followed by cellulitis in $36 \%$ of patients. Gangrene as a feature presented in $14 \%$ of patients and abscess presented in $8 \%$ of patients. Neuropathy was the commonest presentation in patients $(48 \%)$, followed by vasculopathy $(32 \%)$ and $12 \%$ of cases presented with both complications. The microorganism commonly grown on culture of pus was staphylococcus aureus in 11 patients followed by Streptococcus 8, pseudomonas 3, E coli 1 and Proteus1.

In the current study, a vast most majority of patients fell in the age group of 41-50 years; the males outnumbering females and patients mostly developed ulcers within 10-15 years following the diagnosis of diabetes mellitus. The contributing factors are diabetic neuropathy, peripheral arterial disease leading to peripheral vasculopathy and/or any occupations involving outdoor exposure particularly in males [6].

As per studies, male sex have found to be at a significantly higher risk for developing nonhealing ulcer. Also as per observations, the mortality rate is higher in males compared to females when they have poor blood sugars control associated with multi-drug resistant Gram-negative bacilli infecting the diabetic foot and ulcer. Also another significant and a worrying feature was that development of foot ulcer preceded the diagnosis of Type 2 diabetes mellitus in $5.4 \%$ of the included patients. This may be due to the lack of knowledge among the population regarding the symptoms of the disease and proper foot care [7]. Wound cultures showed monomicrobial flora and polymicrobial flora IN THE CURRENT study. These features are identical with similar studies. The current study reveals the increased incidence of monomicrobial flora compared to other studies which may be attributed to probable high prevalence of lesser degree ulcers which may be termed as mild or superficial. The polymicrobial flora routinely colonizes the uninfected ulcers as per many observations. However, in the current study, swabbing for samples was done by curetting from ulcer base instead of suberficial swabbing, and the results yielded many infective ulcer cases, probably pointing towards another cause for exhibiting an increases percentage of singular yield of organisms from the ulcers, There was bacterialfree growth from ulcers in $10.8 \%$ of cases which may be attributable to pre- treatment with antibiotics in majority of cases $(94.6 \%)$ [8].

Our current study highlighted the fact that number of organisms for each ulcer is 1.76 which very well collated with the studies of Zubair et al., [9] and Raja et al., [10]. The organism count per ulcer depends on many factors like ulcer gradation, prior treatment with antibiotics, interval between presentation and development of ulcer or techniques related to sample taking from base or superficially of the ulcer. However compared to current study, various other studies revealed ulcers with increased number of isolates. Our study outcome may also have been influenced by cases with history of cases taking antibiotics prior to study.

The anaerobic bacteria isolation can go upto $51.56 \%$ as seen in many studies but our study showed a lesser isolation of anaerobic bacteria comparatively. The sampling method, history of previous antibiotic therapy and severity or grade of wound has an effect on rate of isolation of Anaerobe. It has been observed that Aerobic bacteria (Staphylococcus spp., Streptococcus spp., and Enterobacteriaceae) are the predominant organisms present in superficial grades of ulcer (Wagner 1 and 2) whereas Anaerobic bacteria are the majority prevalent group in Wagner's Grade 3,4 and 5 ulcers. In the current study by us, majority of Anaerobes isolated were from Grade 4 to Grade 5 ulcer cases and among the isolated Anaerobes, commonest were Bacteroides, and Peptostreptococcus was the second common isolate. These findings are synonymous with the other studies that have postulated Bacteroides sap as the predominant and most common Anaerobe associated with Diabetic Foot ulcers.

\section{CONCLUSION}

The management of Diabetic ulcers should be a multipronged approach which involves the usage of appropriate empirical antibiotic depending upon the wound severity/grading and local antimicrobial susceptibility patterns. The current study highlights the 
fact that $\mathrm{S}$. aureus was the commonest bacterial etiology of Diabetic foot ulcer. The grading and severity of Diabetic ulcers also reveals the number count and type of pathogens infecting Diabetic Foot. As anaerobic bacteria are predominant in Grades 4 and 5 ulcers, it becomes very significant and imperitive to cover these organisms as well in designing a treatment plan. Also poor prognosis of Diabetic foot ulcers are increasingly recognised due to association with multidrug resistance organisms. In selection of an appropriate antibiotic, for diabetic ulcers exhibiting less severity or of superficial grade, Linezolid may be initiated, whereas for advanced or high grade ulcers, Metronidazole, Linezolid and Imipenem can be used. As emphasized earlier, patient education about foot care, appropriate screening measures, early detection and adequate appropriate treatment of Diabetic foot goes a long way in preventing complications and mortalities associated with Diabetic Foot.

\section{REFERENCES}

1. Hefni A, Ibrahim AM, Attia K, Moawad M, Elramah A, Shahin M, Al-Molla M, Al-Satar L. Bacteriological study of diabetic foot infection in Egypt. Journal of the Arab Society for Medical Research. 2013 Jun;8(1):26-32.

2. Chantelau EA. A Novel Diagnostic Test for EndStage Sensory Failure Associated With Diabetic Foot Ulceration: Proof-of-Principle Study. Journal of diabetes science and technology. 2020 Jan 16:1932296819900256.

3. Singh N. MD; David G. Armstrong, DPM, MSc, PhD. Benjamin A. Lipsky, MD. Preventing Foot Ulcers in Patients With Diabetes. JAMA. 2005;293(2):217-28.
4. International Working Group on the Diabetic Foot (2015). "Guidance on the diagnosis, prognosis and management of peripheral artery disease in patients with foot ulcers in diabetes". Retrieved 23 November 2015.

5. Darlington C, Kumar, S, Jagdish S, Sridhar M. Evaluation of Serum Vitamin D Levels in Diabetic Foot Infections: A Cross-Sectional Study in a Tertiary Care Center in South India. Iranian Journal of Medical Sciences, 2019; 44(6):474-482.

6. Criado E, De Stefano AA, Keagy BA, Upchurch Jr GR, Johnson Jr G. The course of severe foot infection in patients with diabetes. Surgery, gynecology \& obstetrics. 1992 Aug 1;175(2):13540.

7. Prompers L, Schaper N, Apelqvist J, Edmonds M, Jude E, Mauricio D, Uccioli L, Urbancic V, Bakker K, Holstein P, Jirkovska A. Prediction of outcome in individuals with diabetic foot ulcers: focus on the differences between individuals with and without peripheral arterial disease. The EURODIALE Study. Diabetologia. 2008 May;51(5):747-55.

8. Shakil S, Khan AU. Infected foot ulcers in male and female diabetic patients: a clinicobioinformative study. Annals of clinical microbiology and antimicrobials. 2010 Dec;9(1):1-10.

9. Zubair M, Malik A, Ahmad J. Clinicobacteriology and risk factors for the diabetic foot infection with multidrug resistant microorganisms in north India. Biol Med. 2010;2(4):22-34.

10. Raja NS. Microbiology of diabetic foot infections in a teaching hospital in Malaysia: A retrospective study of 194 cases. J Microbiol Immunol Infect. 2007;40:39-44. 